\title{
家族性额外小染色体的发现及初步研究
}

\author{
董兆文汪安琦
}

(中国科学院遗传研究所)

Smith 等 ${ }^{[1]}$ 首次报道了一例家族性额外小染色体. 而后, 一些研究者又报道了类似现象, 并对额外小染色体的起源及意义做了探讨 ${ }^{[2-5]}$. 作者在群体细胞遗传学的调查中,发现了一例 带有额外小染色体的家族，在其三代人中发现六名成员带有相同的额外小染色体。应用多种 染色体显带技术,对额外小染色体的性质进行了研究,并讨论了可能起源及意义. 现将研究的 初步结果报道于下.

\section{材料和方 法}

1. 族情况 所研究的家族是一健康的农民家庭，成员达 24 人. 对其中 18 名成员进 行了细胞遗传学研究, 发现 6 名成员带有额外小染色体,其他成员的核型均为正常. 经一般医 学检查, 在所研究的 18 名对象中未发现任何异常. 6 名额外小染色体携带者的一般情况见表 1 .

表 1 六名擭带者的一般情况

\begin{tabular}{|c|c|c|c|c|c|c|}
\hline 编 号 & 性 别 & 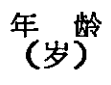 & 与先证者关系 & 型 & 表 & 型 \\
\hline II-2 & 女 & 65 & 母 & $47, X X,+\operatorname{mar}$ & 正 & 常 \\
\hline III-2 & 男 & 41 & 兄 & $47, X Y,+\operatorname{mar}$ & 正 & 常 \\
\hline III-7 & 男 & 36 & 先 证 者 & $47, X Y,+\operatorname{mar}$ & 正 & 常 \\
\hline III-9 & 女 & 29 & 妹 & $47, \mathrm{XX},+\mathrm{mar}$ & 正 & 常 \\
\hline IV -4 & 男 & 6 & 子 & $47, X Y,+\operatorname{mar}$ & 正 & 常 \\
\hline IV -5 & 男 & 5 & 外 & $47, X Y,+$ mar & 正 & 常 \\
\hline
\end{tabular}

2. 实验方法 外周血淋巴细胞培养及染色体制备按常规方法进行. $Q$ 带显示采用 $0.005 \%$ 芥子喹叫因 ( $\mathrm{QM}$ ) 染色. G 带显示采用 $0.2 \%$ 胰酶处理. C 带显示采用 $5 \% \mathrm{Ba}(\mathrm{OH})_{2}$ 处理. 银染色 (Ag-NOR) 采用 $50 \% \mathrm{AgNO}_{3}$ 一步处理法. G 带与银染的复合染色方法由作 者建立, 将另文介绍.

每个被检者计数 30-50 个中期细胞以确定染色体数, 并除外嵌合情况. 选 3-5 个中期 细胞进行核型分析. 显带后的核型分析采用国际命名体制的统一标准 ${ }^{[6]}$.

本文 1981 年 11 月 4 日收到. 


\section{结果和讨 论}

所研究的家系与 18 名研究对象的核型分析结果见图 1.6名额外小染色体携带者是: II2、III-2、III-7、III-9、IV-4、IV-5，其核型均为 $47, \mathrm{XY}$ 或 XX, +mar，未见到嵌合情况. G

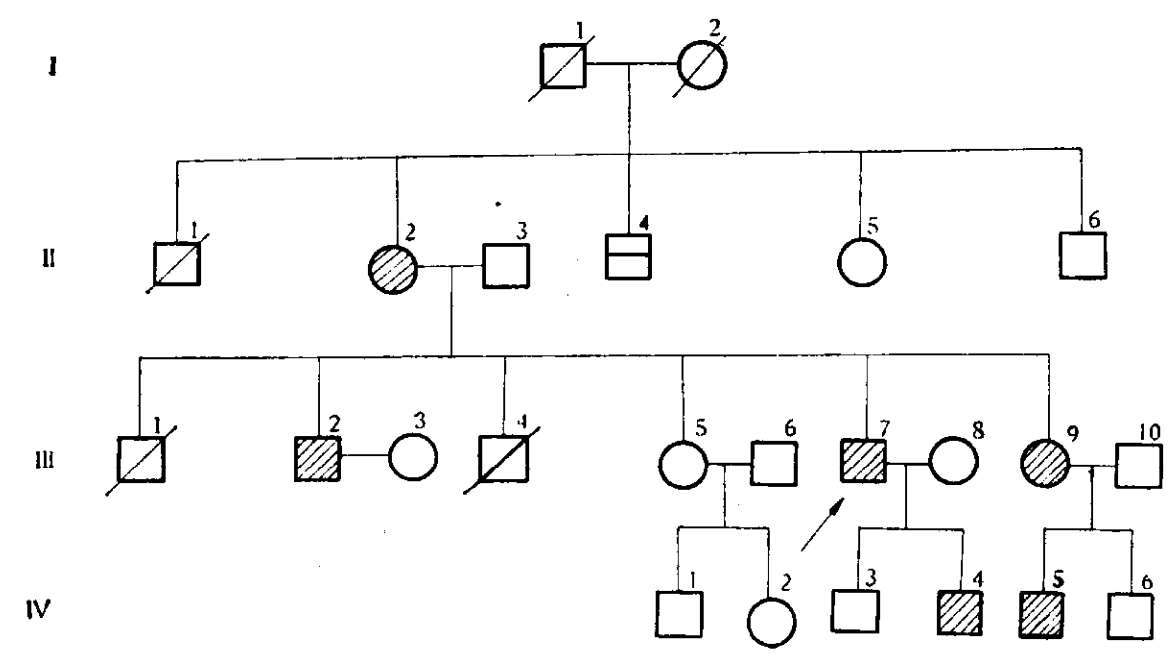

图 1 所研究的家系

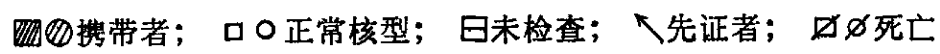

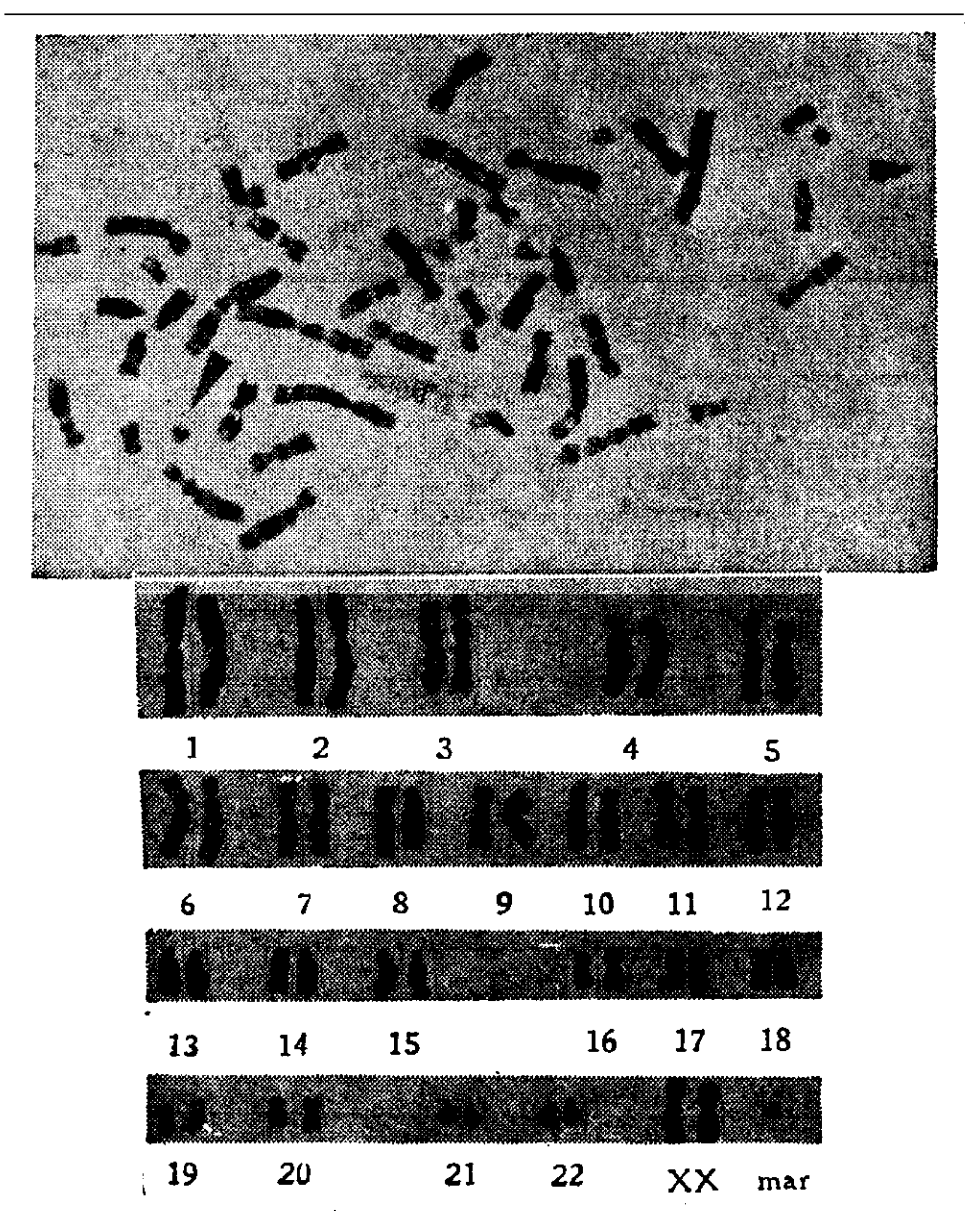

图 $2 \mathrm{G}$ 带处理后的中期细胞与核型 $(47, \times \times,+$ mar $)$

箭头处为额外小染色体 (Trypsin) 
带处理后的中期细胞及核型见图 2. 额外小染色体略小于 G组染色体, 其它染色体未发现异 常.

额外小染色体的各种显带结果见图 3.

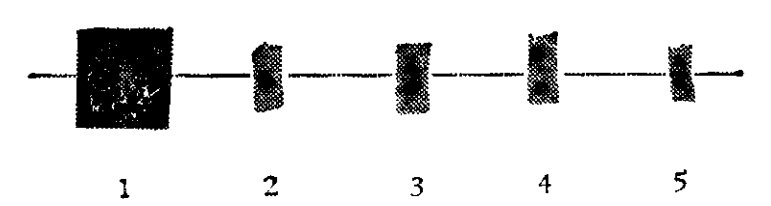

图 3 额外小染色体的各种显带

1. $\mathrm{Q}$ 带；2.G带；3. C带；4.银染色 (Ag-NOR)； $5 . \mathrm{G}$ 带与银染的复合染色

其带型特征为:

$\mathrm{Q}$ 带: 短臂上有一中等强度的荧光带, 长臂上的荧光带较弱,着丝粒部位为阴性.

$\mathrm{G}$ 带: 在长臂与短臂上各有一带, 长臂上的带较短臂上的带为宽. 短臂末端可见随体, 着 丝粒部位着色浅。

C 带：在染色体中部可见两个大小不同的 C 带区.

银染色: 在染色体两端各见一银染区, 二者形态有所不同.

G 带与银染的复合染色：在染色体两端各有一银染区,在一侧有一带,对侧无.

由以上显带结果分析, 本研究中所观察到的额外小染色体是具有双随体和双着丝粒的亚 中部着丝粒染色体.

自 Smith 等首次报道家族性额外小染色体以来, 至今已见 10 篇类似报道. 现将不同作

表 2 不同作者与本文研究结果

\begin{tabular}{|c|c|c|c|c|c|c|}
\hline 参考文献 & 先证者核型 & 先证者表型 & 亲代㩑带者 & 携带者人数 & 携带者表型 & 研究方法 \\
\hline$[1]$ & $47, X Y,+\operatorname{mar}$ & 不 育 & -+ & 二代中 2 人 & 正常 & 未 显带 \\
\hline [2] & $47, X Y,+\operatorname{mar}$ & 智力障碍 & +- & 二代中 2 人 & 癫㾁 & 未显带 \\
\hline$[7]$ & $48, \mathrm{XX},+21,+\operatorname{mar}$ & $\begin{array}{l}\text { Down's } \\
\text { 综 合 征 }\end{array}$ & -+ & 二代中 6 人 & 正常 & 未显带 \\
\hline [3] & $47, \mathrm{XY},+\operatorname{mar}$ & 智力障碍 & -+ & 三代中 6 人 & 正常 & 未显带 \\
\hline [8] & $47, \mathrm{XY},+\operatorname{mar}$ & 正 常 & +- & 二代中 2 人 & 正常 & Q 带 \\
\hline$[4]$ & $47, \mathrm{XX},+\operatorname{mar}$ & 正 常* & -+ & 三代中 6 人 & 正常 & Q 带 \\
\hline [9] & $47, \mathrm{XY},+\operatorname{mar}$ & 正 常* & -+ & 二代中 7 人 & 正常 & \\
\hline$[5]$ & $47, \mathrm{XX},+\operatorname{mar}$ & 正 常** & $-\quad+$ & 二代中 5 人 & 正常 & $Q$ 带, G 带,银染 \\
\hline$[10]$ & $48, x x,+21,+\operatorname{mar}$ & $\begin{array}{l}\text { Down's } \\
\text { 综 合 征 }\end{array}$ & -+ & 二代中 5 人 & 正常*** & $G$ 带, C 带, 银染 \\
\hline$[11]$ & $47, X Y,+\operatorname{mar}$ & 常 & +- & 二代中 2 人 & 正常 & G带, C 带, 银染 \\
\hline 本研究 & $47, \mathrm{XY},+\operatorname{mar}$ & 正 常 & -+ & 三代中 6 人 & 正常 & $\begin{array}{l}Q \text { 带, } G \text { 带, } C \text { 带, 银染, } \\
G \text { 带与银染复合 }\end{array}$ \\
\hline
\end{tabular}

+: 携带者, 一: 正常核型，*先证者是新生儿，**在羊水细胞培养中发现，*** 先证者一妹，其核型为 48， $\mathrm{xx},+21$, +mar. 
者的研究结果与本研究结果列于表 2.

从表 2 中所列结果可知, 各个作者所研究的对象和所用研究方法有所不同. 与其它研究 相比,本研究所发现的家族较大, 研究额外小染色体所用的技术较为全面. 在该家族的三代人 中有 6 名成员带有相同的额外小染色体, 且携带者的表型均为正常, 在文献中较为少见. 经多 种显带技术分析, 额外小染色体的性质比较明确.

在家族性额外小染色体的研究中, 对于额外小染色体的起源, 各个作者根据自己的实验结 果提出不同的推论. 一些学者认为: 额外小染色体是 $D$ 组或 $G$ 组染色体的短臂所形成的等臂 染色体 ${ }^{[2,8]}$; 也有学者认为: 额外小染色体是 $D$ 组或 $G$ 组染色体短臂的易位所致 ${ }^{[5,7]}$. 作者认为 不同研究者所研究对象不同,所观察到的额外小染色体可能是各不相同的,应对各个情况分别 加以分析.

从本研究结果分析, 在该家族中所观察到的额外小染色体, 两端都带有随体, 这说明该染 色体是由 $D$ 组或 $G$ 组染色体的短臂所形成. $Q$ 带与 $G$ 带结果都显示出该染色体两臂明显不对 称. 银染色、 $\mathrm{G}$ 带与银染的复合染色结果也显示出不对称性. 基于上述分析, 本研究中额外小 染色体的起源, 属于等臂染色体的可能性不大, 而可能是 $D / G$ 染色体短臂的易位. 因该染色 体有两个 C 带区, 表明参予易位的染色体短臂都可能包括着丝粒.

在本研究中, 6 名额外小染色体的携带者表型均正常,这与多数研究结果相近. 对于携带 者的表型问题, Armendares 等 ${ }^{[7]}$ 认为: 近端着丝粒染色体的核仁组织者区含有重复性 DNA 序列,具有多套顺反子. 因而在部分缺失的情况下,并不产生表型效应. Romain 等 ${ }^{[5]}$ 的看法是 额外小染色体在遗传活性上可能是情性的, 一般无表型效应. 作者认为: 从本研究结果以及 其它作者在新生儿群体中所做的研究分析, 无表型效应的家族性额外小染色体对研究人类进 化问题可能具有某种意义,值得进一步探讨.

致谢：本工作中得到周宪庭、汤火顺同志的帮助,特此致谢.

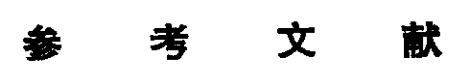

[1] Smith, K. D. et al., Cytogenetics, 4 (1965), 219-226.

[ 2 ] Mukerjee, D. \& Burdette, W. J., Amer. J. Hum. Genet., 18 (1966), 62-69.

[ 3 ] Hoehn, H. et al., Cytogenetics, 9 (1970), 186-198.

[ 4 ] Friedrich, U. \& Nielsen, J., Clinical Genetics, 6 (1974), 23-31.

[5] Romain, D. R. et al., Clinical Genetics, 16 (1979), 183-190.

[6] An International System for Human Cytogenetic Nomenclature, Cytogenet. Cell Genet., 21 (1978), 309-404.

[ 7 ] Armondares, s. et al., Cytogenetics, 8 (1969), 177-186.

[ 8 ] Nielsen, J. \& Hreidarsson, A. B., Humangenetit, 19 (1973), 271-274.

[9] Walzer, S. \& Gerald, P. S., in Population Cytogenetics Studies in Human (Eds. Hook, E. B. \& Porter. I. H.), Acad. Press, 1977.

[10] Ramos. C. et al., Hum. Genet., 49 (1979), 7-10.

[11] Howard-Peebles, P. N., The Journal of Heredity, 70 (1979), 347-348. 